\title{
DETERMINING SPECIFIC THYROID TRANSCRIPTS IN PERIPHERAL BLOOD: A SINGLE CENTER STUDY EXPERIENCE
}

\author{
Makazlieva $\mathrm{T}^{1, \#, *}$, Eftimov $\mathrm{A}^{2, \#}$, Vaskova $\mathrm{O}^{1}$, Tripunoski $\mathrm{T}^{1}$, \\ Miladinova $\mathrm{D}^{1}$, Risteski $\mathrm{S}^{3}$, Jovanovic $\mathrm{H}^{3}$, Jakovski $\mathrm{Z}^{3}$ \\ \#Tanja Makazlieva and Aleksandar Eftimov contributed equally to this study \\ and are considered first coauthors.
}

*Corresponding Author: Tanja Makazlieva, Ph.D., Institute of Pathophysiology and Nuclear Medicine, Medical Faculty, Mother Teresa Street, No. 17, 1000, Skopje, Republic of Macedonia.

Mobile: +389-75-313-665. E-mail: tmakazlieva@medf.ukim.edu.mk or tmakazlieva@gmail.com

\begin{abstract}
Thyroid carcinoma (TC) comprises a spectrum of different tumors with a wide range of biological behavior and prognosis. The techniques based on the latest trends in molecular biology may have application in diagnosis of metastatic TC. The aim of this study was to apply and analyze mRNA expression in peripheral blood of thyrotropin receptor [thyroid stimulating hormone receptor (TSHR-mRNA)] gene and thyroglobulin ( $T g$-mRNA) gene using $2^{-\Delta \Delta C t}$ method in differentiated TC patients and healthy individuals. Fifty-seven subjects were included in the study, consisting of 40 patients with TC and 17 healthy volunteers as a control group. Total RNA was isolated from peripheral blood and used for two-step reverse transcriptase-polymerase chain reaction (PCR). Real-time PCR was performed with appropriate primers. Relative quantification using the $2^{-\Delta \Delta \mathrm{Ct}}$ method was applied. Thyroid carcinoma patients with metastatic disease or loco-regional relapse expressed TSHR-mRNA by a 8.57-fold higher level than healthy controls. Thyroid carcinoma patients with biochemical relapse expressed TSHRmRNA by a 14.17 -fold higher level than healthy controls, while expression of $T g$-mRNA was 6.6-fold higher in TC patients with metastatic disease and loco-regional relapse than healthy controls and 8.34-fold higher level compared with TC patients with excellent response to treatment. Our

\footnotetext{
${ }^{1}$ Institute of Pathophysiology and Nuclear Medicine, Medical Faculty, Skopje, Republic of Macedonia

${ }^{2}$ Laboratory of Molecular Pathology, Institute of Pathology, Medical Faculty, Skopje, Republic of Macedonia

${ }^{3}$ Institute for Forensic Medicine, Criminology and Medical Deontology, Medical Faculty, Skopje, Republic of Macedonia
}

preliminary study showed that the TSHR gene expression might have more useful application as a biomarker compared to detection of $\mathrm{Tg}$ gene expression.

Keywords: Thyroglobulin $(\mathrm{Tg})$ gene expression; Thyroid carcinoma (TC); Thyroid stimulating hormone receptor (TSHR) gene expression.

\section{INTRODUCTION}

Thyroid carcinoma (TC) comprises a spectrum of different tumors with a wide range of biological behavior and prognosis [1-3]. Differentiated thyroid carcinomas (DTC) including papillary thyroid carcinoma (PTC) and follicular thyroid carcinomas (FTC) originate from follicular epithelial cells. The progression of DTC is usually slow, but local involvement of lymph nodes is very often possible, as well as loco-regional relapses as late as 30 years after the initial diagnosis. In some cases, distant metastatic disease is present even at initial presentation of the disease [4-6]. The recommendations for long-term follow-up of DTC usually include ultrasound of the neck, serum thyroglobulin (sTg) levels and radioiodine whole body scan ( ${ }^{131} \mathrm{I}$ WBS). The sTg level is a very useful marker in detecting progression of the disease, but this analysis has no diagnostic value in patients with coexisting autoimmune thyroid disease and positive anti-thyroglobulin antibodies (aTg). Endogenous aTg may cause false-negative results in immunometric assays for sTg determination, and sTg levels in those patients may not reveal the exact state of the disease [7].

According to the new guidelines, in some patients with microcarcinomas and low-risk tumors, the radioiodine ablation (RAI) is not recommended, leading to a situation where it is inconvenient to apply sTg as a tumor marker, imposing 
the need for new biomarkers of the disease [8]. Experiences with diagnostic detection of circulating tumor cells in patients with some solid tumors as breast, colon and prostate carcinomas, are a motivation for researchers to apply similar methodology in TC [9]. For this purpose, the techniques based on the latest trends in molecular biology can have the most appropriate application in diagnosis of metastatic TC.

The use of the real-time polymerase chain reaction (RT-PCR) method for detection of circulating malignant thyroid cells was first introduced by Ditkoff et al. [10] evaluating $T g$-mRNA transcripts in peripheral blood in both healthy individuals and patients with TC, which yielded promising results, leading to an increased number of published papers on this topic in the years to come. The reports about possible the usefulness of this approach in different studies vary greatly [11]. The main objective of this study was to apply and analyze gene expression of thyreotropin receptor [thyroid stimulating hormone receptor (TSHRmRNA)] gene and thyroglobulin $(\mathrm{Tg})$ gene in peripheral blood of healthy individuals and patients with DTC, using RT-PCR and relative quantification with the $2^{-\Delta \Delta C t}$ method.

\section{MATERIALS AND METHODS}

Subjects. The study included blood sampling from a total of 57 subjects, including 40 patients with DTC and 17 healthy volunteers as a control group. The inclusion criteria for the control group were normal isoechoic appearance of the thyroid gland and absence of nodules at ultrasound examination (US) of the neck (linear transducer 7.5-10 MHz), normal blood levels for thyroid stimulating hormone (TSH) and free thyroxine $\left(\mathrm{FT}_{4}\right)$, and antithyroid antibodies that are below the level usually considered to be clinically significant. In all patients from the DTC group, the surgery and ablation treatment with radioactive ${ }^{131} \mathrm{I}$ were conducted at least 6 months before starting the research. In all DTC patients blood samples for RNA extraction were drawn prior to US neck examination. Similar to the concept of risk stratification during follow up introduced by Tuttle et al. [12], DTC patients were divided in three subgroups. The three groups of patients were selected according to blood levels of sTg (CRM 457, Immulite 2000; Siemens, Munich, Bavaria, Germany) and aTg, as well as on the US neck examination and findings from WBS after ablation with ${ }^{131} \mathrm{I}$ or diagnostic WBS. The first group consisted of patients with incomplete structural response to treatment and biochemical relapse of the disease, imaging confirmed the metastatic spread or loco-regional relapse and elevated sTg levels, $>0.2 \mathrm{ng} / \mathrm{mL}$ (TCs 22 patients). The second group were patients with incomplete biochemical response and indeterminate response, with only elevated $\operatorname{sTg}(>0.2$ $\mathrm{ng} / \mathrm{mL}$ ), or elevated aTg levels, without confirmed and known morphological signs for relapse (TCb six patients). The third group of patients were with complete structural and biochemical response to therapy, excellent responders (TCr 12 patients). Corresponding to the histopathological type from 40 patients, four cases were FTC, 25 typical variants of PTC, one PTC in thyroglossal duct cyst, one papillary microcarcinoma, seven follicular variants of PTC and two Hurtle cell carcinomas. The study was approved by the Ethics Committee at the Medical Faculty of the University "Ss Cyril and Methodius," Skopje, Republic of Macedonia, and written consent was obtained from all subjects enrolled in the research.

RNA Extraction Procedure and Real Time-Polymerase Chain Reaction. Whole blood samples were drawn from HC and TC patients in standard $3 \mathrm{~mL}$ EDTA blood vacutainers, total RNA was extracted using commercially available RNA isolation kit GenElute ${ }^{\mathrm{TM}}$ Total RNA purification kit (Sigma Aldrich Co. LLC, St. Louis, MO, USA) and isolated total RNA was used for two step RTPCR with ReadyScript ${ }^{\mathrm{TM}}$ cDNA Synthesis Mix kit (Sigma Aldrich), according to the manufacturer's protocols.

Real Time Polymerase Chain Reaction. The PCR step was performed using the following primer pairs: TSHR-F 5'-GCT TTT CAG GGA CTA TGC AAT GAA3' and TSHR-R 5'-AAG GGC AGT GAC ACT GGT TTG AGA-3', targeted to amplify a segment spanning exons 6 to 9 (nucleotides 555-767 or 212 bp) and Tg- F 5'-AGG GAA ACG GCC TTT CTGAA-3' and Tg-R 5'-GTG GAGAAG ACG ACG ATT TC-3', targeted to exons 1 to 5 (nucleotides $112-519$ or $407 \mathrm{bp}$ ) [9]. The ubiquitously expressed GAPDH gene was used to confirm RNA extraction and RT-PCR using primers GAPDH-F 5'-TTC GTC ATG GGT GTG AAC C-3' and GAPDH-R 5'-GAT GAT GTT CTG GAG AGC CC-3', as previously reported [13,14]. For RT quantitative PCR (qPCR), we used Hot FirePol Eva Green qPCR Mix Plus (Rox) PCR master mix (Solis BioDyne, Tartu, Estonia). The reaction mixture was incubated at 95 ${ }^{\circ} \mathrm{C}$ for $15 \mathrm{~min}$., followed by 38 cycles of denaturation at 95 ${ }^{\circ} \mathrm{C}$ for 15 seconds, annealing at $62{ }^{\circ} \mathrm{C}$ for 20 seconds and elongation at $72{ }^{\circ} \mathrm{C}$ for 20 seconds, for $T S H R$ and $T g$ and incubated at $95{ }^{\circ} \mathrm{C}$ for $15 \mathrm{~min}$., followed by 38 cycles of denaturation at $95{ }^{\circ} \mathrm{C}$ for 15 seconds, annealing at $57{ }^{\circ} \mathrm{C}$ for 20 seconds and elongation at $72{ }^{\circ} \mathrm{C}$ for 20 seconds, for $G A P D H$. All samples were analyzed in triplicate.

Relative Quantification Method. Relative quantification was applied by calculating fold change in gene expression of the TSHR and $T g$ target genes, normalized to the endogenous reference gene GAPDH. Cycle threshold $(\mathrm{Ct}), \Delta \mathrm{Ct}, \Delta \Delta \mathrm{Ct}$ and normalized relative expression ratio values, were calculated according to the $2^{-\Delta \Delta \mathrm{Ct}}$ method, by Livak and Schmittgen [15]. According to this method, $\Delta \mathrm{Ct}_{\mathrm{TSHR}}=\left(\right.$ average $\mathrm{Ct}_{\mathrm{TSHR}}$-average $\left.\mathrm{Ct}_{\mathrm{GAPDH}}\right)$, also for 
$\Delta \mathrm{Ct}_{\mathrm{Tg}}=$ (average $\mathrm{Ct}_{\mathrm{Tg}}$-average $\mathrm{Ct}_{\mathrm{GAPDH}}$ ) for every $\mathrm{TC}$ patient and $\mathrm{HC}$ was calculated, and then $\Delta \Delta \mathrm{Ct}=[\Delta \mathrm{Ct}(\mathrm{TC})-$ $\Delta \mathrm{Ct}(\mathrm{HC})]$ was calculated from the average values of four groups and later normalized expression ratio $2^{-\Delta \Delta \mathrm{Ct}}$ was applied for fold change evaluation [15,16]. Statistically significant differences among all groups were evaluated with the Mann-Whitney U and Kruskal-Wallis H tests.

\section{RESULTS}

In our study, expression for the GAPDH gene was detected in all 57 samples. From all 57 samples, seven TSHR and/or $T g$ expression levels were undetectable, three were from $\mathrm{HC}$ (in two cases both target genes were undetectable in peripheral blood, and in one only $T g$-mRNA was undetectable). From the patients included in the examination, one from the TCb group had undetectable $T g$-mRNA, only one from the TCs group had undetectable levels for both target genes, and two other patients with advanced stages of the disease had undetectable TSHR-mRNA. The highest and lowest mean values of $\Delta \mathrm{Ct}=\left(\mathrm{Ct}_{\mathrm{TSHR}}-\mathrm{Ct}_{\mathrm{GAPDH}}\right)$ was in $\mathrm{TCr}$ and TCs, respectively $(12 \pm 1.8$ vs. 9.6 \pm 2.6$)$. The statistical analysis revealed significant difference between the mean values $\Delta \mathrm{Ct}=\left(\mathrm{Ct}_{\mathrm{TSHR}}-\mathrm{Ct}_{\mathrm{GAPDH}}\right)$ for all four groups $\left(\chi^{2}=13.312 ; \mathrm{df}=3, p=0.004\right)$ (Figure 1 and Table 1). Additional intergroup analysis of the mean $\Delta \mathrm{Ct}=\left(\mathrm{Ct}_{\mathrm{TSHR}}{ }^{-}\right.$ $\left.\mathrm{Ct}_{\mathrm{GAPDH}}\right)$ revealed significant difference between: a) TCs/ $\operatorname{TCr}(Z=-2.231 ; p=0.026) ; b) \mathrm{TCs} / \mathrm{HC}(\mathrm{Z}=-3.416 ; p=$ $0.0006)$; $) \mathrm{TCb} / \mathrm{TCr}(Z=-2.341 ; p=0.019)$ and $\mathrm{d}) \mathrm{TCb} /$ $\mathrm{HC}(\mathrm{Z}=-2.803 ; p=0.005)$ (Table 2$)$.

We have analyzed the statistical significance among values of $\Delta \mathrm{Ct}=\left(\mathrm{Ct}_{\mathrm{TSHR}}-\mathrm{Ct}_{\mathrm{GAPDH}}\right)$ between two groups of

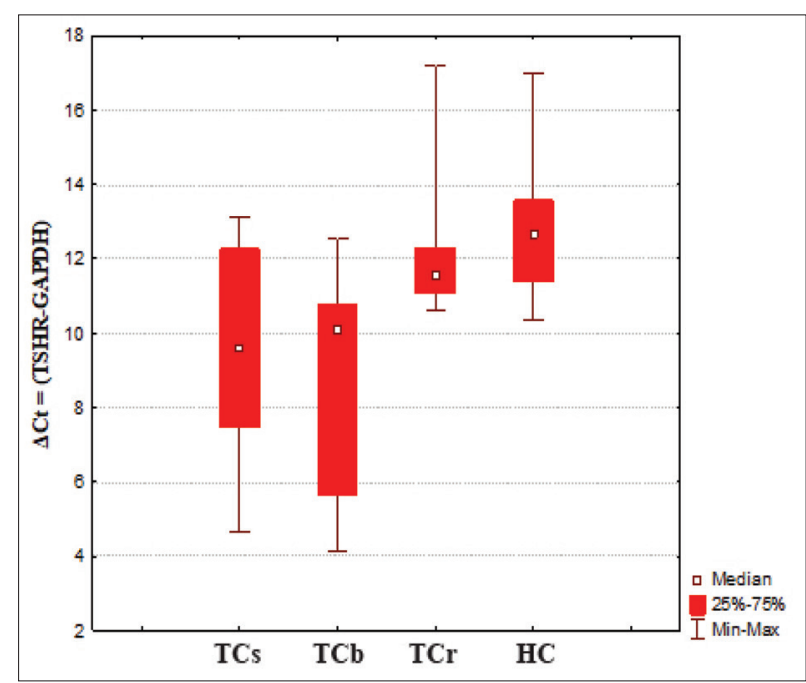

Figure 1. Distribution of $\Delta \mathrm{Ct}=\left(\mathrm{Ct}_{\mathrm{TSHR}}-\mathrm{Ct}_{\mathrm{GAPDH}}\right)$ among four evaluated groups (see tables).

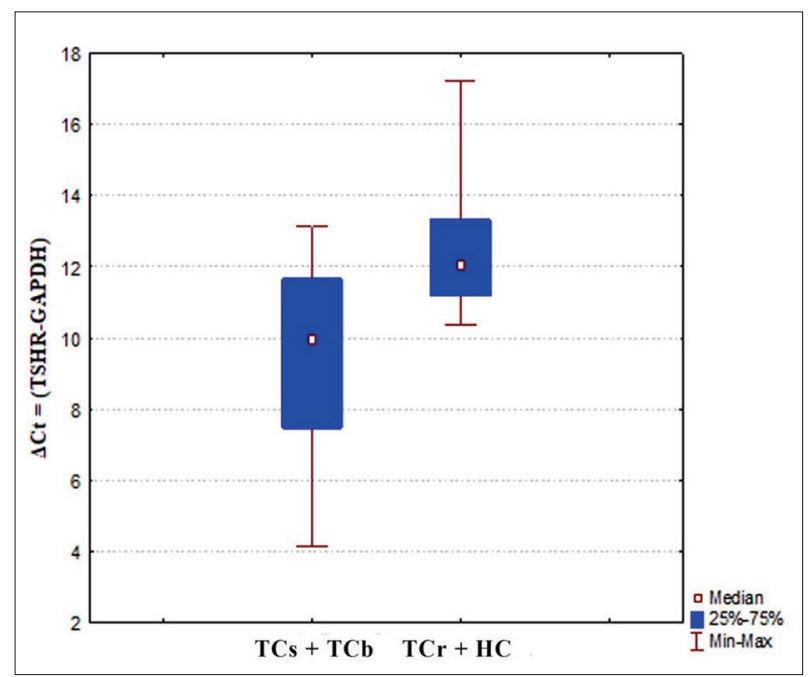

Figure 2. Two-group comparison for $\Delta \mathrm{Ct}=\left(\mathrm{Ct}_{\mathrm{TSHR}}-\mathrm{Ct}_{\mathrm{GAPDH}}\right)$ values (see tables).

Table 1. Four intergroup comparisons for $\Delta \mathrm{Ct}=\left(\mathrm{Ct}_{\mathrm{TSHR}}-\mathrm{Ct}_{\mathrm{GAPDH}}\right)$ values.

\begin{tabular}{|l|c|c|c|c|c|c|c|c|}
\hline Groups & Mean & $n$ & SD & Minimum & Maximum & \multicolumn{3}{|c|}{ Percentiles } \\
\hline & & & & & & $25^{\text {th }}$ & $50^{\text {th }}($ median) & $75^{\text {th }}$ \\
\hline $\mathrm{TCs}^{\mathrm{a}}$ & 9.62 & 19 & 2.66 & 4.66 & 13.13 & 7.47 & 9.58 & 12.26 \\
\hline $\mathrm{TCb}^{\mathrm{b}}$ & 8.89 & 6 & 3.26 & 4.15 & 12.55 & 5.63 & 10.13 & 10.77 \\
\hline $\mathrm{TCr}^{\mathrm{c}}$ & 12.04 & 12 & 1.76 & 10.59 & 17.21 & 11.06 & 11.55 & 12.29 \\
\hline $\mathrm{HC}^{\mathrm{d}}$ & 12.72 & 15 & 1.65 & 10.36 & 17.00 & 11.39 & 12.66 & 13.57 \\
\hline Summary & 10.99 & 52 & 2.70 & 4.15 & 17.21 & 10.13 & 11.21 & 12.57 \\
\hline
\end{tabular}

SD: standard deviation. Kruskal-Wallis H test: $\zeta^{2}=13.312 ; \mathrm{df}=3 ; p=0.004$. Statistical significance: $p<0.05$.

a TCs: patients with incomplete structural response to treatment and biochemical relapse.

${ }^{\mathrm{b}} \mathrm{TCb}$ : patients with incomplete biochemical response to treatment.

${ }^{c} \mathrm{TCr}$ : patients with complete structural response and biochemical response, excellent response.

${ }^{\mathrm{d}} \mathrm{HC}$ : healthy controls. 


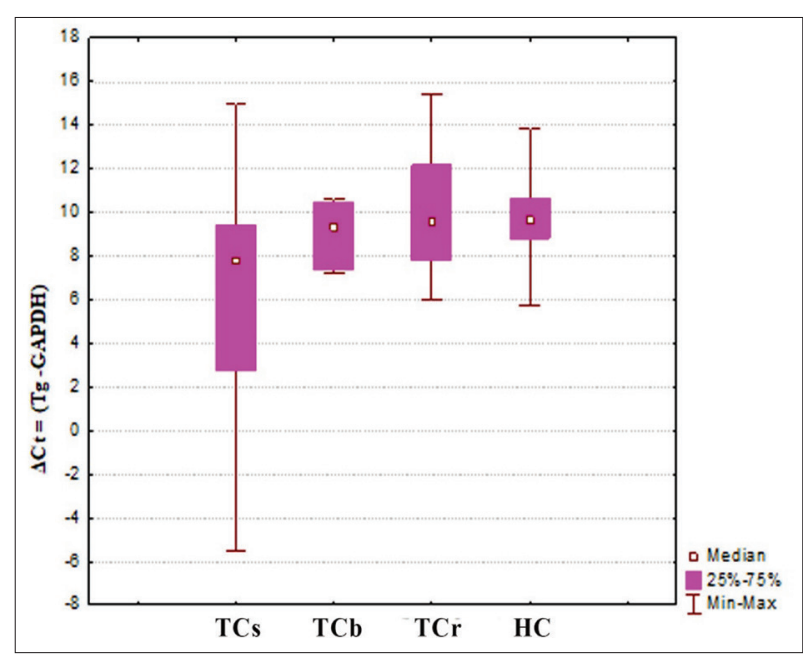

Figure 3. Four-group comparison for $\Delta \mathrm{Ct}=\left(\mathrm{Ct}_{\mathrm{Tg}}-\mathrm{Ct}_{\mathrm{GAPDH}}\right)$ values (see tables).

patients with incomplete response to treatment $(\mathrm{TCs}+\mathrm{TCb})$ and two groups composed of patients with excellent response to treatment and healthy individuals $(\mathrm{TCr}+\mathrm{HC})$. Analysis revealed significant differences between the two combined groups $(Z=-3.928 ; p=0.00008)$ (Table 3 and Figure 2).

Within the four research intergroups, comparison for mean $\Delta \mathrm{Ct}=\left(\mathrm{Ct}_{\mathrm{Tg}}-\mathrm{Ct}_{\mathrm{GAPDH}}\right)$ values was also performed. The highest and lowest mean value for $\Delta \mathrm{Ct}=\left(\mathrm{Ct}_{\mathrm{Tg}}-\mathrm{Ct}_{\mathrm{GAPDH}}\right)$

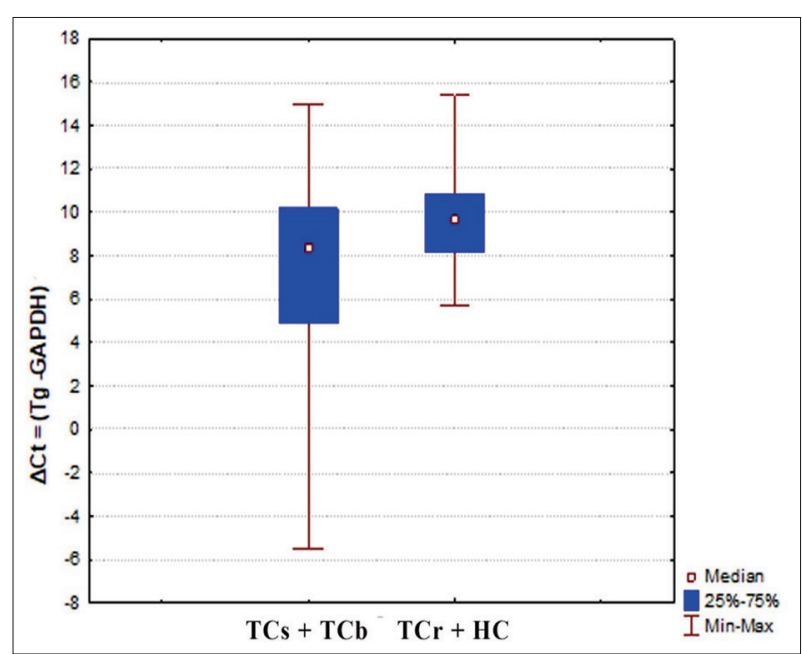

Figure 4. Two-group comparison of $\Delta \mathrm{Ct}=\left(\mathrm{Ct}_{\mathrm{Tg}}-\mathrm{Ct}_{\mathrm{GAPDH}}\right)$ (see tables).

was detected in the TCr group and TCs with $10 \pm 2.6$ vs. $6.17 \pm 5.37$, respectively (Table 4 and Figure 3 ).

Further intergroup analysis related to mean values of $\Delta \mathrm{Ct}=\left(\mathrm{Ct}_{\mathrm{Tg}}-\mathrm{Ct}_{\mathrm{GAPDH}}\right)$ revealed statistical significant differences between: a) TCs/TCr $(Z=-1.964 ; p=0.049)$; b) TCs $/ \mathrm{HC}(Z=-2.453 ; p=0.014)$ (Table 5). Within the research comparison between two combined groups for mean $\Delta \mathrm{Ct}=\left(\mathrm{Ct}_{\mathrm{Tg}}-\mathrm{Ct}_{\mathrm{GAPDH}}\right)$ values was performed (Table 6 and Figure 4).

Table 2. Intergroup paired comparison in $\Delta \mathrm{Ct}=\left(\mathrm{Ct}_{\mathrm{TSHR}}-\mathrm{Ct}_{\mathrm{GAPDH}}\right)$ values.

\begin{tabular}{|l|c|c|c|c|c|c|}
\hline Mann-Whitney U Test & $\mathbf{T C s}^{\mathbf{a}} / \mathbf{T C b}^{\mathbf{b}}$ & $\mathbf{T C s}^{\mathbf{a}} / \mathbf{T C r}$ & $\mathbf{T C s}^{\mathbf{a}} / \mathbf{H C}^{\mathbf{d}}$ & $\mathbf{T C b}^{\mathbf{b}} / \mathbf{T C} \mathbf{r}^{\mathbf{c}}$ & $\mathbf{T C b}^{\mathbf{b}} / \mathbf{H C}^{\mathbf{d}}$ & $\mathbf{T C r}^{\mathbf{c}} / \mathbf{H C}^{\mathbf{d}}$ \\
\hline Z value & 0.382 & -2.231 & -3.416 & -2.341 & -2.803 & -1.757 \\
\hline Asymptotic significance (two-tailed) & 0.703 & $0.026^{\mathrm{e}}$ & $0.0006^{\mathrm{e}}$ & $0.019^{\mathrm{e}}$ & $0.005^{\mathrm{e}}$ & 0.079 \\
\hline
\end{tabular}

a TCs: patients with incomplete structural response to treatment and biochemical relapse.

${ }^{\mathrm{b}} \mathrm{TCb}$ : patients with incomplete biochemical response to treatment.

c TCr: patients with complete structural response and biochemical response, excellent response.

${ }^{\mathrm{d}} \mathrm{HC}$ : healthy controls.

e Statistical significance $p<0.05$.

Table 3. Two-group comparison for $\triangle \mathrm{Ct}=(T S H R-G A P D H)$ values.

\begin{tabular}{|l|c|c|c|c|c|c|c|c|}
\hline Groups & Mean & $n$ & SD & Minimum & Maximum & \multicolumn{3}{|c|}{ Percentiles } \\
\hline & & & & & & $25^{\text {th }}$ & $50^{\text {th }}($ median $)$ & $75^{\text {th }}$ \\
\hline $\mathrm{TCs}^{\mathrm{a}}+\mathrm{TCb}^{\mathrm{b}}$ & 9.44 & 25 & 2.76 & 4.15 & 13.13 & 7.465 & 9.94 & 11.66 \\
\hline $\mathrm{TCr}^{\mathrm{c}}+\mathrm{HC}^{\mathrm{d}}$ & 12.42 & 27 & 1.70 & 10.36 & 17.21 & 11.20 & 12.05 & 13.33 \\
\hline Summary & 10.99 & 52 & 2.70 & 4.15 & 17.21 & 10.12 & 11.21 & 12.57 \\
\hline
\end{tabular}

SD: standard deviation; Mann-Whitney U test: $\mathrm{Z}=-3.928 ; p=0.00008$. Statistical significance: $p<0.05$.

a TCs: patients with incomplete structural response to treatment and biochemical relapse.

${ }^{\mathrm{b}} \mathrm{TCb}$ : patients with incomplete biochemical response to treatment.

c TCr: patients with complete structural response and biochemical response, excellent response.

${ }^{\mathrm{d}} \mathrm{HC}$ : healthy controls. 
Table 4. Four-group comparison for $\Delta \mathrm{Ct}=\left(\mathrm{Ct}_{\mathrm{Tg}}-\mathrm{Ct}_{\mathrm{GAPDH}}\right)$ values.

\begin{tabular}{|l|c|c|c|c|c|c|c|c|}
\hline Groups & Mean & $n$ & SD & Minimum & Maximum & \multicolumn{3}{|c|}{ Percentiles } \\
\hline & & & & & & $25^{\text {th }}$ & $50^{\text {th }}($ median) & $75^{\text {th }}$ \\
\hline $\mathrm{TCs}^{\mathrm{a}}$ & 6.17 & 21 & 5.37 & -5.49 & 15.00 & 2.74 & 7.79 & 9.40 \\
\hline $\mathrm{TCb}^{\mathrm{b}}$ & 9.07 & 6 & 1.55 & 7.22 & 10.61 & 7.39 & 9.36 & 10.47 \\
\hline $\mathrm{TCr}^{\mathrm{c}}$ & 10.02 & 11 & 2.60 & 6.01 & 15.45 & 7.82 & 9.62 & 12.17 \\
\hline $\mathrm{HC}^{\mathrm{d}}$ & 9.68 & 16 & 2.28 & 5.71 & 13.85 & 8.81 & 9.69 & 10.64 \\
\hline Summary & 8.32 & 54 & 4.16 & 5.49 & 15.45 & 7.23 & 8.86 & 10.37 \\
\hline
\end{tabular}

SD: standard deviation; Kruskal-Wallis H test: $\zeta^{2}=7.9481 ; \mathrm{df}=3 ; p=0.0471$. Statistical significance: $p<0.05$.

${ }^{a}$ TCs: patients with incomplete structural response to treatment and biochemical relapse.

b TCb: patients with incomplete biochemical response to treatment.

${ }^{c}$ TCr: patients with complete structural response and biochemical response, excellent response.

${ }^{\mathrm{d}} \mathrm{HC}$ : healthy controls.

Table 5. Intergroup paired comparison in $\Delta \mathrm{Ct}=\left(\mathrm{Ct}_{\mathrm{Tg}}-\mathrm{Ct}_{\mathrm{GAPDH}}\right)$ values.

\begin{tabular}{|l|c|c|c|c|c|c|}
\hline Mann-Whitney U Test & $\mathbf{T C s}^{\mathbf{a}} / \mathbf{T C b}^{\mathbf{b}}$ & $\mathbf{T C s}^{\mathbf{a}} / \mathbf{T C} \mathbf{r}^{\mathbf{c}}$ & $\mathbf{T C s}^{\mathbf{a}} / \mathbf{H C}^{\mathbf{d}}$ & $\mathbf{T C b}^{\mathbf{b}} / \mathbf{T C} \mathbf{r}^{\mathbf{c}}$ & $\mathbf{T C b}^{\mathrm{b}} / \mathbf{H C}^{\mathbf{d}}$ & $\mathbf{T C r}^{\mathbf{c}} / \mathbf{H C}^{\mathbf{d}}$ \\
\hline Z value & -1.166 & -1.964 & -2.453 & -0.402 & -0.442 & 0.148 \\
\hline Asymptotic significance (two-tailed) & 0.243 & $0.049^{\mathrm{e}}$ & $0.014^{\mathrm{e}}$ & 0.688 & 0.658 & 0.882 \\
\hline
\end{tabular}

a TCs: patients with incomplete structural response to treatment and biochemical relapse.

${ }^{\mathrm{b}} \mathrm{TCb}$ : patients with incomplete biochemical response to treatment.

c TCr: patients with complete structural response and biochemical response, excellent response.

${ }^{\mathrm{d}} \mathrm{HC}$ : healthy controls.

${ }^{\mathrm{e}}$ Statistical significance $p<0.05$.

Table 6. Two-group comparison for $\mathrm{Ct}=\left(\mathrm{Ct}_{\mathrm{Tg}}-\mathrm{Ct}_{\mathrm{GAPDH}}\right)$ values.

\begin{tabular}{|l|c|c|c|c|c|c|c|c|}
\hline Groups & Mean & $n$ & SD & Minimum & Maximum & \multicolumn{3}{|c|}{ Percentiles } \\
\hline & & & & & & $25^{\text {th }}$ & $50^{\text {th }}($ median $)$ & $75^{\text {th }}$ \\
\hline $\mathrm{TCs}^{\mathrm{a}}+\mathrm{TCb}^{\mathrm{b}}$ & 6.81 & 27 & 4.91 & -5.49 & 15.00 & 4.915 & 8.35 & 10.17 \\
\hline $\mathrm{TCr}^{\mathrm{c}}+\mathrm{HD}^{\mathrm{d}}$ & 9.82 & 27 & 2.53 & 5.71 & 15.45 & 8.14 & 9.62 & 10.85 \\
\hline Summary & 8.32 & 54 & 4.16 & -5.49 & 15.45 & 7.23 & 8.86 & 10.37 \\
\hline
\end{tabular}

SD: standard deviation; Mann-Whitney U test: $Z=-2.413 ; p=0.0158$. Statistical significance: $p<0.05$.

a TCs: patients with incomplete structural response to treatment and biochemical relapse.

${ }^{\mathrm{b}} \mathrm{TCb}$ : patients with incomplete biochemical response to treatment.

${ }^{c}$ TCr: patients with complete structural response and biochemical response, excellent response.

${ }^{\mathrm{d}} \mathrm{HC}$ : healthy controls.

Table 7. Fold change in expression of TSHR and $T g$ target genes between TCs and HC/TCr groups and between $\mathrm{TCb}$ and $\mathrm{HC} / \mathrm{TCr}$ groups.

\begin{tabular}{|l|c|c|}
\hline Fold Change & For TSHR-mRNA & For $\boldsymbol{T g}$-mRNA \\
\hline$\Delta \Delta \mathrm{Ct}=\Delta \mathrm{Ct}(\mathrm{TCs})-\Delta \mathrm{Ct}(\mathrm{HC}) 2^{-\Delta \Delta \mathrm{Ct}}$ & $-3.09904(\mathbf{8 . 5 7})$ & $-2.72137(\mathbf{6 . 6})$ \\
\hline$\Delta \Delta \mathrm{Ct}=\Delta \mathrm{Ct}(\mathrm{TCs})-\Delta \mathrm{Ct}(\mathrm{TCr}) 2^{-\Delta \Delta \mathrm{Ct}}$ & $-2.42523(\mathbf{5 . 3 7})$ & $-3.06057(\mathbf{8 . 3 4})$ \\
\hline$\Delta \Delta \mathrm{Ct}=\Delta \mathrm{Ct}(\mathrm{TCb})-\Delta \mathrm{Ct}(\mathrm{HC}) 2^{-\Delta \Delta \mathrm{Ct}}$ & $-3.82478(\mathbf{1 4 . 1 7})$ & $-0.61184(\mathbf{1 . 5 3})$ \\
\hline$\Delta \Delta \mathrm{Ct}=\Delta \mathrm{Ct}(\mathrm{TCb})-\Delta \mathrm{Ct}(\mathrm{TCr}) 2^{-\Delta \Delta \mathrm{Ct}}$ & $-3.15097(\mathbf{8 . 8 8})$ & $-0.95104(\mathbf{1 . 9 3})$ \\
\hline
\end{tabular}

a TCs: patients with incomplete structural response to treatment and biochemical relapse.

${ }^{\mathrm{b}} \mathrm{TCb}$ : patients with incomplete biochemical response to treatment.

${ }^{c}$ TCr: patients with complete structural response and biochemical response, excellent response.

${ }^{\mathrm{d}} \mathrm{HC}$ : healthy controls. 
Using the $2^{-\Delta \Delta \mathrm{Ct}}$ method, according to the previously described quantification by Livak and Schmittgen [14], we detected that TCs patients expressed TSHR-mRNA by a 8.57-fold higher level than HC individuals. The TCs patients expressed TSHR by a 5.37-fold higher level than TCr patients, TCb patients expressed TSHR by a 14.17 -fold higher level than HC individuals, and TCb patients expressed TSHR by an 8.88-fold higher level than TCr patients (Table 7). Whereas for $T g$-mRNA, we found a 6.6-fold higher change in expression in the TCs group compared to HC, an 8.34-fold higher change in expression in the TCs group than $\mathrm{TCr}$, a 1.53-fold change in expression in $\mathrm{TCb}$ and $\mathrm{HC}$, and a 1.93fold change in expression in TCb compared to TCr (Table 7).

\section{DISCUSSION}

Since Ditkoff et al. [10] reported a possible usefulness of the RT-PCR molecular technique in detection of thyroid circulating cells in blood samples as an indicator of metastatic TC, an increased number of studies on this issue have been published in the past few years [17-19]. Besides the possible use in follow-up of TC, some researchers reported that evaluating $T S H R-\mathrm{mRNA}$ in patients with indeterminate cytology, reports from fine needle aspiration biopsies of thyroid nodules might improve cancer detection and avoid unnecessary surgeries [20,21].

Using data from previous investigations into relative quantification of gene expression in other malignancies, we decided to perform a relative gene expression using the $2^{-\Delta \Delta \mathrm{Ct}}$ method in evaluating relative expression of $\mathrm{Tg}$ and TSHR genes in patients with TC compared with the HC group. The analysis revealed an 8.57-fold higher level in TCs patients than in $\mathrm{HC}$ individuals, and TCb patients expressed TSHR by a 14.17-fold higher level than HC individuals. The increase in fold change in expression was higher for $\mathrm{TCb}$ with incomplete biochemical response than in the TCs group, compared to HC subjects $[11,14,15]$. This finding may be due to dedifferentiation of tumor in the TCs group of patients and low expression of the evaluated transcripts for the target genes.

In our study, in almost all HC (except three cases) expression of both $T g$ and TSHR genes was detected. Most of the studies found the presence of $T g$ and TSHR expression in blood of normal subjects [20-22]. This finding can be explained due to the presence of transcripts for $T g$ and TSHR from thyrocytes in peripheral blood of HC or from ectopically transcribed $T g$ and $T S H R$, which may indicate a low specificity of this method, but we detected statistically significant lower expression in HC compared to the TCs group.

Eszlinger et al. [22] analyzed the usefulness of quantification of only $T g$-mRNA in peripheral blood in the follow-up of DTC patients. Their analysis differed from ours because they did not find any statistically significant difference in expression of $T g$-mRNA in patients with and without metastatic disease; hence, they concluded that evaluation of $T g$-mRNA was not a useful biomarker in the follow-up of TC patients [22].

Ringel et al. [23] analyzed the possible reasons for great variations in results between different researchers and found several possible reasons, starting from the number of cycles, selection of primers, selection of "housekeeping genes" and difference in endpoint, or quantification of the products in the assay or simply absence in standardization of methodology [23]. Bojunga et al. [24] and Takano et al. [25] in their studies didn't find statistical significant difference in expression levels between patients with and without metastases. Bojunga et al. [24] reported different results using "normal vs. high sensitivity PCR technique." After 30 cycles of PCR, they detected $T g$-mRNA in 9/13 patients with known metastases, in half of the patients without metastases and in 21/85 patients with benign disease. After using 40 cycles of PCR, $T g$-mRNA expression was increased in 11/13 patients with metastases as well as in $61 / 85$ patients with benign disease and in 41/50 healthy controls [24].

Another important question regarding this methodology, that should be discussed, is careful selection of the primer pairs. Gupta et al. [13] in their research, used several primer pairs of different exons. They found that primer pairs for TSHR targeting 6 to 9 exons and 1 to 5 exons for $T g$, were with specificity for thyroid tissue and no reactivity in normal peripheral blood mononuclear cells excluding possible illegitimate transcription $[13,14]$. In our study, we used the same primer pairs as in the study of Gupta et al. [13]. On the other hand, Savagner et al. [26] also evaluated the possible effect of alternative splicing using two different primer pairs from two non overlapping regions. First in exons 10 and 11, in which no alternative splicing was described, second in exons 6 and 7, in which alternative splicing was found. Their study revealed higher expression levels in controls depending on the TSH level, even higher than in patients with metastatic disease, but low levels in patients after thyroidectomy and ablation and without signs of disease persistence. These authors found that alternative variants of $T g$-mRNA represented approximately $30.0 \%$ of the measured value of $T g$-mRNA in controls and patients [25].

In our study, we noted significant difference in expressions of both target genes between patients with structural and biochemical incomplete response to treatment compared to healthy individuals, and this finding was more remarkable for expression of TSHR-mRNA. Our study differs from the previous study in endpoint quantification of the methodology. 
Savagner et al. [26] also used absolute quantification, creating a standard curve from serial dilutions $10^{2}-10^{5}$ copies of $T g$ cDNA, generated from plasmids containing appropriate cDNA inserted as a template and using a cutoff value for TC patients' values above $1 \mathrm{pg} T g$-mRNA/ $\mu \mathrm{g}$ RNA. These authors found mean $10.6 \pm 3.1 \mathrm{pg} T g-\mathrm{mRNA} / \mu \mathrm{g}$ total RNA in normal healthy control individuals [26]. Chinnappa et al. [14] analyzed the presence of TSHR-mRNA and TgmRNA in 51 normal subjects, 67 patients with DTC, 27 patients with benign thyroid disease and eight patients with DTC preoperatively. They used a similar methodology to ours (38 cycles, GAPDH as reference gene and the same primer pairs for $T g$ and $T S H R$ ), except for the method of quantification. For the endpoint results, they used 2.0\% gel electrophoresis and visualization of the products with ethidium bromide staining. The results of this study showed absence of TSHR-mRNA and $T g$-mRNA in blood samples of normal subjects, which is contrary to what we found; furthermore, they presented $97.0 \%$ sensitivity for TSHRmRNA and $94.0 \%$ sensitivity for $T g$-mRNA based on the results obtained from patients with and without known metastatic disease and from benign group [14].

In our study, we detected the presence of TSHRmRNA and $T g$-mRNA in 14/17 (82.4\%) of HC and in all TC patients, except in three patients with elevated sTg levels, loco-regional persistence of the disease in one patient and distant metastasis in two other patients. In two patients, high levels of $\mathrm{sTg}>300 \mathrm{ng} / \mathrm{mL}$ were found, as well as confirmed metastatic disease, in one patient with diffuse pulmonary secondary deposits and several ${ }^{131}$ I therapies and in the other one with skeletal deposits. Statistical analysis revealed significant difference in $\Delta \mathrm{Ct}$ value for expression of $\mathrm{Tg}$ and TSHR genes between TCs and $\mathrm{TCb}$ patients and $\mathrm{TCr}$-excellent therapy responders and HC. The greatest difference was detected between the TCb and HC groups for TSHR, and lower, but statistically significant difference between TCs and HC. This finding may be due to the more aggressive variants of tumors included in the TCs group and possible dedifferentiation of the tumor. We also found that TSHR-mRNA might be a more precise biomarker in follow-up of TC than $T g$-mRNA. Discrepancy in the results obtained in many studies indicate that a larger multicenter study including more subjects and standardization of the methodology used, especially in quantification, is needed for understanding the real significance of this method. Alternative splicing, illegitimate transcription, empirically supposed $100.0 \%$ of amplification efficiency and dedifferentiation of the tumor are also possible reasons for errors in the detection of transcripts. The real need of new biomarkers in the follow-up of thyroid carcinomas exists, especially after introduction of the new recommendations for ratio- nalization of ${ }^{131} \mathrm{I}$ ablation treatment in low risk DTC and microcarcinomas, thus, sTg levels could not be used as a reliable tumor marker in the follow-up of these patients.

\section{CONCLUSIONS}

Data from our analysis revealed expression of TSHR and $\mathrm{Tg}$ transcripts both in TC patients with incomplete response to treatment and in $\mathrm{HC}$ controls and TC patients with excellent response to treatment. We found statistically significant difference in $\Delta \mathrm{Ct}$ value for both target genes. The TCs patients expressed TSHR-mRNA by a 8.57 -fold higher level than HC individuals and 6.6-fold higher for $\mathrm{Tg}$-mRNA. The highest fold change of 14.17 in expression of TSHR-mRNA was found between the TCb group and HC. Our preliminary study showed significant difference in TSHR-mRNA and Tg-mRNA expression levels between TCs patients and HC and TCr group of patients. Further studies with a larger number of subjects and standardization of the methodology, especially in quantification, are needed for understanding the real significance of this method.

Declaration of Interest. The authors report no conflicts of interest. The authors alone are responsible for the content and writing of this article.

\section{REFERENCES}

1. Li Volsi VA. Papillary thyroid carcinoma: An update. Mod Pathol. 2011; 24(2): S1-S9.

2. Pellegriti G, Frasca F, Regalbuto C, Squatrito S, Vigneri R. Worldwide increasing incidence of thyroid cancer: Update on epidemiology and risk factors. J Cancer Epidemiol. 2013; 2013: 965212.

3. Lloyd RV, Buehler D, Khanafshar E. Papillary thyroid carcinoma variants. Head Neck Pathol. 2011; 5(1): 51-56.

4. De Groot LJ, Reed Larsen P, Hennemann G, Editors. The Thyroid and Its Diseases, 6th ed. New York, NY, USA: Churchill Livingstone, 1996.

5. Burns WR, Zeiger MA. Differentiated thyroid cancer. Semin Oncol. 2010; 37(6): 557-66.

6. Verburg FA, Lips CJM, Lentjes EGWM, De Klerk $\mathrm{JMH}$. Detection of circulating Tg-mRNA in the follow-up of papillary and follicular thyroid cancer: how useful is it? Br J Cancer. 2004; 91(2): 200-204.

7. Schlumberger M, Pacini F, Tuttle RM, Editors. Thyroid Tumors, 4th ed. Paris, France: Institute MedicoEducatif, 2015. 
8. Haugen BR, Alexander EK, Bible KC, Doherty GM, Mandel SJ, Nikiforov YE, et al. 2015 American Thyroid Association Management Guidelines for Adult Patients with Thyroid Nodules and Differentiated Thyroid Cancer. The American Thyroid Association Guidelines Task Force on Thyroid Nodules and Differentiated Thyroid Cancer. Thyroid. 2016; 26(1): 1-133.

9. Andergassen U, Zebisch M, Kölbl AC, König A, Heublein S, Schröder L, et al. Real-time qPCR-based detection of circulating tumor cells from blood samples of adjuvant breast cancer patients: A preliminary study. Breast Care. 2016; 11(3):194-198.

10. Ditkoff BA, Marvin MR, Yemul S, Shi YJ, Chabot J, Feind C, et al. Detection of circulating thyroid cells in peripheral blood. Surgery. 1996; 120(6): 959-964; discussion 964-965.(?)

11. Ringel MD. Molecular detection of thyroid cancer: Differentiating "signal" and "noise" in clinical assays. J Clin Endocrinol Metab. 2004; 89(1): 29-32.

12. Tuttle RM, Tala H, Shah J, Leboeuf R, Ghossein R, Gonen $\mathrm{M}$, et al. Estimating risk of recurrence in differentiated thyroid cancer after total thyroidectomy and radioactive iodine remnant ablation: using response to therapy variables to modify the initial risk estimates predicted by the new American Thyroid Association staging system. Thyroid. 2010; 20(12): 1341-1349.

13. Gupta MK, Taguba L, Arciaga R, Siperstein A, Faiman C, Mehta A, et al. Detection of circulating thyroid cells by reverse transcription-PCR for thyroid-stimulating hormone receptor and thyroglobulin: the importance of primer selection. Clin Chem. 2002; 48(10): 1862-1865.

14. Chinnappa P, Taguba L, Arciaga R, Faiman C, Siperstein A, Mehta AE. Detection of thyrotropin-receptor messenger ribonucleic acid (mRNA) and thyroglobulin mRNA transcripts in peripheral blood of patients with thyroid disease: Sensitive and specific markers for thyroid cancer. J Clin Endocrinol Metab. 2004; 89(8):3705-3709.

15. Livak KJ and Schmittgen TD. Analysis of relative gene expression data using real-time quantitative PCR and the $2^{-\triangle \Delta C T}$ method. Methods. 2001; 25(4):402-408.

16. Rao X, Huang X, Zhou Z, Lin X. An improvement of the $2^{\text {-delta deltact }}$ method for quantitative real-time polymerase chain reaction data analysis. Biostat Bioinforma Biomath. 2013; 3(3):71-85.
17. Badulescu IC, Barbus E, Piciu D. Circulating tumor cells in thyroid carcinoma - The prognostic role of this biomarker. Review of the literature. Clujul Med. 2017; 90(3):256-261.

18. Barzon L, Boscaro M, Paenti M, Taccaliti A, Palu G. Evaluation of circulating thyroid-specific transcripts as markers of thyroid cancer relaps. Int J Cancer. 2004; 110(6): 914-920.

19. Wingo ST, Ringel MD, Anderson JS, Patel AD, Lukes YD, Djuh YY, et al. Quantitative reverse trans-cription-PCR measurement of thyroglobulin mRNA in peripheral blood of healthy subjects. Clin Chem. 1999; 45(6 Pt 1):785-789.

20. Teama SH, Agwa SHA, Fawzy A, Sayed MM, Ibrahim WA, Eid YM. Molecular detection of circulating thyroid specific transcripts (TSHR/Tg-mRNAs) in thyroid cancer patients: Their diagnostic significance. Egypt J Med Hum Genet. 2011; 12(2): 201-209.

21. Gupta M, Chia SY. Circulating thyroid cancer markers. Curr Opin Endocrinol Diabetes Obes. 2007; 14(5): 383-388.

22. Eszlinger M, Neumann S, Otto L, Paschke R. Threoglobulin mRNA quantification in the peripheral blood is not a reliable marker for the follow-up of the patients with differentiated thyroid cancer. Eur J Endocrinol. 2002; 147(5): 575-582.

23. Ringel MD, Balducci-Silano PL, Anderson JS, Spencer CA, Silverman J, Sparling YH, et al. Quantitative reverse transcription-PCR of circulating thyroglobulin messenger ribonucleic acid for monitoring patients with thyroid carcinoma. J Clin Endocrinol Metab. 1999; 84(11): 4037-4042.

24. Bojunga J, Röddiger S, Stanisch M, Kusterer K, Kurek R, Renneberg $\mathrm{H}$, et al. Molecular detection of thyroglobulin mRNA transcripts in peripheral blood of patients with thyroid disease by RT-PCR. Br J Cancer. 2000; 82(10): 1650-1655.

25. Takano T, Miyauchi A, Yoshida H, Hasegawa Y, Kuma K, Amino N. Quantitative measurement of thyroglobulin mRNA in peripheral blood of patients after total thyroidectomy. Br J Cancer. 2001; 85(1): 102-106.

26. Savagner F, Rodien P, Reynier P, Rohmer V, Bigorgne JC, Malthiery Y. Analysis of Tg transcripts by real time RT PCR in blood of thyroid cancer patients. J Clin Endocrinol Metab. 2002; 87(2): 635-639. 